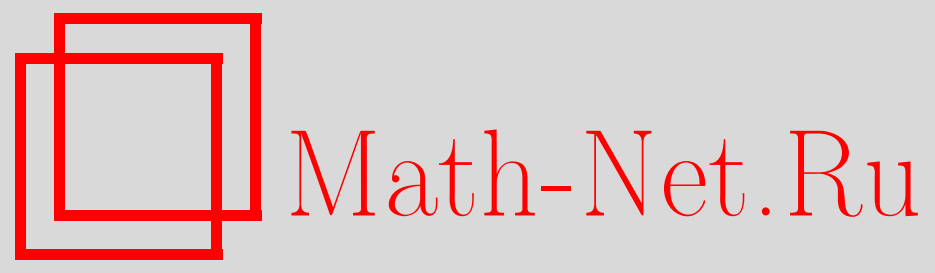

А. Н. Алексейчук, Условия однозначности проблемы моментов в классе q-распределений, Дискрет. матем., 1999, том 11, выпуск 4, 48-57

DOI: https://doi.org/10.4213/dm391

Использование Общероссийского математического портала Math-Net.Ru подразумевает, что вы прочитали и согласны с пользовательским соглашением http://www . mathnet.ru/rus/agreement

Параметры загрузки:

IP : 35.173 .137 .237

26 апреля 2023 г., 17:04:32

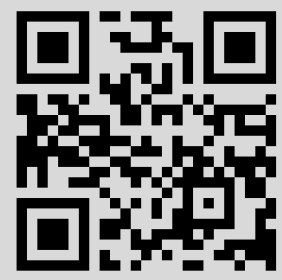




\title{
Условия однозначности проблемы моментов в классе $q$-распределений
}

\author{
(c) 1999 г. А. Н. Алексейчук
}

Пусть $K_{q}-$ класс распределений вероятностей, сосредоточенных на множестве степеней числа $q>1$ с неотрицательными целыми показателями ( $q$-распределений), $\mathbf{P}=\left\{p_{k}=P\left(q^{k}\right), k=0,1, \ldots\right\}$ - распределение вероятностей из класса $K_{q}$, обладающее моментами любого порядка. Показано, что для того, чтобы распределение $\mathrm{P}$ однозначно определялось в классе $K_{q}$ последовательностью своих моментов при условии, что $p_{k}>0, k=0,1, \ldots$, необходимо, а при условии

$$
\sup _{k \rightarrow \infty} \lim _{k}\left(p_{k} q^{\left(\begin{array}{c}
k \\
2
\end{array}\right)}\right)^{1 / k}<\infty
$$

достаточно, чтобы имели место соотношения

$$
\inf _{k \rightarrow \infty} \lim _{2 k} q^{\left(\begin{array}{c}
2 k \\
k
\end{array}\right)}=\inf _{k \rightarrow \infty} \lim _{2 k+1} q^{\left(\begin{array}{c}
2 k+1 \\
2
\end{array}\right)}=0 .
$$

Полученные результаты применяются при исследовании предельного распределения числа решений системы случайных линейных однородных уравнений с равновероятной матрицей коэффициентов над конечным локальным кольцом главных идеалов.

\section{1. Основные результаты}

Пусть $\mathbf{P}$ - распределение вероятностей, принадлежащее классу $K_{q}$ распределений, сосредоточенных на множестве $\left\{q^{k}, k=0,1, \ldots\right\}, q>1$. Пусть

$$
p_{k}=\mathbf{P}\left\{q^{k}\right\}, \quad Q_{k}=\sum_{t=k}^{\infty}\left[\begin{array}{l}
t \\
k
\end{array}\right]_{q} p_{t}, \quad k=0,1, \ldots
$$

где

$$
\left[\begin{array}{l}
t \\
k
\end{array}\right]_{q}=\frac{\left(q^{-1}\right)_{t}}{\left(q^{-1}\right)_{k}\left(q^{-1}\right)_{t-k}} q^{k(t-k)}, \quad\left(q^{-1}\right)_{t}=\prod_{i=1}^{t}(1-q)^{-i}, \quad t \in \mathbf{N}_{0}, \quad k=0,1, \ldots, t
$$

(при $t=0$ полагаем $\left(q^{-1}\right)_{t}=1$ ). 
В [1] установлены соотношения

$$
\begin{aligned}
& Q_{k}=\prod_{j=0}^{k-1}\left(q^{k}-q^{j}\right)^{-1} \sum_{l=0}^{k}(-1)^{l} q^{\left(\begin{array}{c}
l \\
2
\end{array}\right)}\left[\begin{array}{l}
k \\
l
\end{array}\right]_{q} m_{k-l}, \\
& m_{k}=\sum_{l=0}^{k}\left[\begin{array}{l}
k \\
l
\end{array}\right]_{q} Q_{l} \prod_{j=0}^{l-1}\left(q^{l}-q^{j}\right), \quad k=0,1, \ldots,
\end{aligned}
$$

между числами $Q_{k}$ и начальными моментами

$$
m_{k}=\sum_{t=0}^{\infty} p_{t} q^{t k}, \quad k=0,1, \ldots,
$$

распределения вероятностей $\mathbf{P}$ и показано, что $\mathbf{P}$ однозначно определяется в классе $K_{q}$ последовательностью своих моментов, если ряд

$$
Q(z)=\sum_{k=0}^{\infty} Q_{k} q^{\left(\begin{array}{c}
k \\
2
\end{array}\right)} z^{k}
$$

сходится в точке $z=1$.

В настоящей работе получены достаточные и близкие к ним необходимые условия однозначности проблемы моментов в классе $K_{q}$ в предположении, что ряд $Q(z)$ имеет ненулевой радиус сходимости.

Обозначим $\theta$ наименьшее неотрицательное целое число, для которого ряд (2) сходится в точке $z=q^{-\theta}$. Описание распределений $\left\{\pi_{k}, k=0,1, \ldots\right\} \in K_{q}$, удовлетворяющих условию

$$
\sum_{t=k}^{\infty}\left[\begin{array}{l}
t \\
k
\end{array}\right]_{q} \pi_{t}=Q_{k}, \quad k=0,1, \ldots,
$$

т. е., в силу (1), имеющих те же начальные моменты, что и распределение $\mathbf{P}$, содержит следующая теорема.

Теорема 1. Пусть $\left\{\pi_{k}, k=0,1, \ldots\right\}$ - распределение вероятностей из класса $K_{q}$, удовлетворяющее условию (3). Тогда для любого $t=\theta, \theta+1, \ldots$ имеет место равенство

$$
\pi_{t}=\sum_{l=0}^{\theta-1} \pi_{l} \sum_{k=l}^{\theta-1} \frac{q^{-\left(\begin{array}{c}
t-k \\
2
\end{array}\right)}(-1)^{t-k}}{\left(q^{-1}\right)_{t-k}\left(q^{-1}\right)_{k-l}}+\sum_{k=0}^{t-\theta} \frac{Q\left(-q^{-(t-k)}\right) q^{-\left(\begin{array}{c}
k \\
2
\end{array}\right)}(-1)^{k}}{\left(q^{-1}\right)_{k}\left(q^{-1}\right)_{t-k}}
$$

В частности, вектор

$$
\left(\pi_{0}, \pi_{1}, \ldots, \pi_{\theta-1}\right)
$$

является неотрицателъным решением системы линейных неравенств

$$
\sum_{l=0}^{\theta-1} x_{l} \sum_{k=l}^{\theta-1} \frac{q^{-\left(\begin{array}{c}
t-k \\
2
\end{array}\right)}(-1)^{t-k}}{\left(q^{-1}\right)_{t-k}\left(q^{-1}\right)_{k-l}}+\sum_{k=0}^{t-\theta} \frac{Q\left(-q^{-(t-k)}\right) q^{-\left(\begin{array}{c}
k \\
2
\end{array}\right)}(-1)^{k}}{\left(q^{-1}\right)_{k}\left(q^{-1}\right)_{t-k}} \geqslant 0, \quad t=\theta, \theta+1, \ldots
$$

Обратно, для любого неотрицательного решения (5) системъ неравенств (6) последовательность $\left\{\pi_{t}, t=0,1, \ldots\right\}$, определяемая с помощью соотношения (4), является распределением вероятностей, удовлетворяющим условию (3). 
Следствие 1. В условиях теоремъ 1 распределение вероятностей $\mathbf{P}$ однозначно определяется своими моментами в классе $K_{q}$ в том и толъко том случае, когда система неравенств (6) имеет единственное неотрицательное решение.

Следующая теорема устанавливает достаточные условия однозначности проблемы моментов в классе $K_{q}$ в терминах вероятностей $p_{k}, k=0,1, \ldots$

Теорема 2. При выполнении соотночений

$$
\begin{gathered}
\sup _{k \rightarrow \infty} \lim _{k}\left(p_{k} q^{\left(\begin{array}{c}
k \\
2
\end{array}\right)}\right)^{1 / 2}<\infty, \\
\inf _{k \rightarrow \infty} \lim _{2 k} p^{\left(\begin{array}{c}
2 k \\
2
\end{array}\right)}=\inf _{k \rightarrow \infty} \lim _{2 k+1} q^{\left(\begin{array}{c}
2 k+1 \\
2
\end{array}\right)}=0
\end{gathered}
$$

распределение $\mathbf{P}$ определяется последовательностъю своих моментов в классе $K_{q}$ однозначно.

В п. 2 показано, что (7) равносильно существованию ненулевого радиуса сходимости ряда (2).

Отметим, что при вылолнении условия

$$
p_{k}>0, \quad k=0,1, \ldots
$$

соотношения (8) необходимо имеют место, если моменты $m_{k}, k=0,1, \ldots$, однозначно определяют распределение вероятностей $\mathbf{P}$.

Действительно, предположим, что, например,

$$
\lim _{k \rightarrow \infty} p_{2 k} q^{\left(\begin{array}{c}
2 k \\
2
\end{array}\right)}>0 \text {. }
$$

Тогда в силу (9) существует $\varepsilon>0$ такое, что

$$
p_{2 k} q^{\left(\begin{array}{c}
2 k \\
2
\end{array}\right)}\left(q^{-1}\right)_{2 k}>\varepsilon
$$

для всех $k=0,1, \ldots$ Используя тождество [2, стр. 32]

$$
\sum_{t=0}^{\infty}(-1)^{t} \frac{q^{-\left(\begin{array}{c}
t \\
2
\end{array}\right)}}{\left(q^{-1}\right)_{t}}=\prod_{t=0}^{\infty}\left(1-q^{-t}\right)=0
$$

находим, что

$$
\sum_{t=k}^{\infty}\left[\begin{array}{l}
t \\
k
\end{array}\right]_{q}\left(p_{t}-\varepsilon \frac{(-1)^{t} q^{-\left(\begin{array}{c}
t \\
2
\end{array}\right)}}{\left(q^{-1}\right)_{t}}\right)=\sum_{t=k}^{\infty}\left[\begin{array}{l}
t \\
k
\end{array}\right]_{q} p_{t}, \quad k=0,1, \ldots,
$$

откуда в силу (1) следует, что распределения вероятностей $\left\{p_{k}, k=0,1, \ldots\right\}$ и $\left\{p_{k}-\varepsilon \frac{(-1)^{k} q^{-\left(\begin{array}{c}k \\ 2\end{array}\right)}}{\left(q^{-1}\right)_{k}}, k=0,1, \ldots\right\}$ обладают одинаковыми начальными моментами.

Несколько более слабое достаточное условие

$$
p_{k} q^{\left(\begin{array}{l}
k \\
2
\end{array}\right)} \geqslant \varepsilon>0, \quad k=0,1, \ldots,
$$

неоднозначности проблемы моментов в классе $K_{q}$ получено И.Н. Коваленко (не опубликовано). 
В качестве приложения результатов сформулированных выше теорем рассмотрим предельное распределение числа решений системы случайных линейных однородных уравнений

$$
A x=0
$$

над конечным локальным кольцом главных идеалов $R$, где $A$-равновероятная матрица размера $(n+s) \times n$ над $R, s-$ фиксированное целое число.

Пусть $|R|=q^{N}, R / J(R)=\mathrm{GF}(q)$, где $J(R)$ - радикал [3, стр.94] кольца $R$. Обозначим $\nu_{n, s}^{(N)}$ - число решений системы линейных уравнений (10). Тогда [4]

$$
\begin{aligned}
& \rho_{s}^{(N)}(k)=\lim _{n \rightarrow \infty} \mathbf{P}\left(\nu_{n, s}^{(N)}=q^{k}\right)=\left(q^{-1}\right)_{\infty} \sum_{r=s_{0}}^{k} q^{-r(s N+k)} \\
& \times \sum_{\left(\beta_{1}, \ldots, \beta_{N}\right) \in B_{r, s, k}} \frac{q^{\sum_{1 \leqslant j<i \leqslant N}(i-j) \beta_{i} \beta_{j}}}{\left(q^{-1}\right)_{\beta_{1}} \ldots\left(q^{-1}\right)_{\beta_{N}}\left(q^{-1}\right)_{\beta_{N-s}}}, \quad k=s_{0} N, s_{0} N+1, \ldots,
\end{aligned}
$$

где

$$
\left(q^{-1}\right)_{\infty}=\prod_{i=1}^{\infty}\left(1-q^{-i}\right), \quad s_{0}=\max \{-s, 0\}
$$

и

$$
\begin{aligned}
B_{r, s, k}=\left\{\left(\beta_{1}, \ldots, \beta_{N}\right) \in \mathbf{Z}^{\mathbf{N}} \mid \beta_{i} \geqslant 0, i=\right. & 1, \ldots, N, \beta_{N} \geqslant s \\
& \left.\sum_{i=1}^{N} \beta_{i}=r+s, \sum_{i=1}^{N-1} \beta_{i}(N-i)=N r-k\right\} .
\end{aligned}
$$

В [5, стр. 167] показано, что распределение вероятностей

$$
\left\{\rho_{s}^{(N)}\left(k+s_{0} N\right), k=0,1, \ldots\right\}
$$

обладает моментами любого порядка.

Теорема 3. Распределение вероятностей (12) однозначно определяется последовательностъю своих моментов в классе $K_{q}$ тогда и толъко тогда, когда $N \leqslant 2$.

Доказательство. Случай $N=1$ рассмотрен в [1]. При $N=2, k=s_{0} N, s_{0} N+1, \ldots$ на основании (11)

$$
\begin{aligned}
\rho_{s}^{(2)}(k) & =\left(q^{-1}\right)_{\infty} \sum_{r=\lceil k / 2\rceil}^{\min \{k, k+s\}} q^{-r(2 s+k)} \frac{q^{(2 r-k)(s+k-r)}}{\left(q^{-1}\right)_{2 r-k}\left(q^{-1}\right)_{s+k-r}\left(q^{-1}\right)_{k-r}} \\
& <\frac{q^{-k s-k^{2}}}{\left(q^{-1}\right)_{\infty}^{2}} \sum_{r=0}^{k} q^{2 r(k-r)} \leqslant \frac{q^{-k s-k^{2}}}{\left(q^{-1}\right)_{\infty}^{2}} q^{-k / 2}(k+1) .
\end{aligned}
$$

Следовательно, при $k=0,1, \ldots$

$$
\rho_{s}^{(2)}\left(k+2 s_{0}\right) q^{\left(\begin{array}{c}
k \\
2
\end{array}\right)}<\frac{k+2 s_{0}+1}{\left(q^{-1}\right)_{\infty}^{2}} q^{-2 s_{0}\left(s+s_{0}\right)} q^{-k / 2-k\left(s+2 s_{0}\right)},
$$


где правая часть стремится к нулю при $k \rightarrow \infty$.

Таким образом, в силу теоремы 2 распределение вероятностей (12) определяется своими моментами в классе $K_{q}$ однозначно.

При $N \geqslant 3, k=s_{0} N, s_{0} N+1, \ldots$, оценим сумму (11) слагаемым, соответствующим значению $r=\lceil k / N\rceil$. В результате получим неравенства

$$
\rho_{s}^{(N)}(k)>\left(q^{-1}\right)^{\infty} q^{-\lceil k / N\rceil(s N+k)}>0,
$$

из которых следует, что $\rho_{s}^{(N)}\left(k+s_{0} N\right) q^{\left(\begin{array}{c}k \\ 2\end{array}\right)} \rightarrow \infty$ при $k \rightarrow \infty$.

Таким образом, соотношения (8) не выполняются, и, следовательно, при $N \geqslant 3$ распределение вероятностей (12) не определяется однозначно последовательностью своих моментов.

\section{2. Доказательство теорем 1 и 2}

Установим ряд вспомогательных утверждений.

Предположим, что ряд (2) сходится в круге $|z|<R$, где $R>0$.

Лемма 1. Для того чтобъ последователъность $\left\{\pi_{k}, k=0,1, \ldots,\right\}$ неотрицательных чисел удовлетворлла условию (3), необходимо и достаточно, чтобы для всех $z \in \mathbf{C},|z|<R$, имело место равенство

$$
Q(z)=\sum_{t=0}^{\infty} \pi_{t} \prod_{i=0}^{t-1}\left(1+q^{i} z\right) .
$$

Доказательство. Докажем необходимость. Поскольку при $|z|<R$ ряд (2) сходится абсолютно, в силу (3) при $|z|<R$ абсолютно сходится двойной ряд

$$
\sum_{k=0}^{\infty} \sum_{t=k}^{\infty}\left[\begin{array}{l}
t \\
k
\end{array}\right]_{q} q^{\left(\begin{array}{c}
k \\
2
\end{array}\right)} z^{k} \pi_{t}
$$

Переставляя члены этого ряда и используя тождество [2, стр. 50]

$$
\sum_{k=0}^{t}\left[\begin{array}{l}
t \\
k
\end{array}\right]_{q} q^{\left(\begin{array}{c}
k \\
2
\end{array}\right)} z^{k}=\prod_{i=0}^{t-1}\left(1+q^{i} z\right)
$$

получаем, что

$$
Q(z)=\sum_{t=0}^{\infty} \pi_{t}\left(\sum_{k=0}^{t}\left[\begin{array}{l}
t \\
k
\end{array}\right]_{q} q^{\left(\begin{array}{c}
k \\
2
\end{array}\right)} z^{k}\right)=\sum_{t=0}^{\infty} \pi_{t} \prod_{i=0}^{t-1}\left(1+q^{i} z\right) .
$$

Докажем достаточность. В силу (13), (14) двойной ряд

$$
\sum_{t=0}^{\infty} \sum_{k=0}^{t} \pi_{t}\left[\begin{array}{l}
t \\
k
\end{array}\right]_{q} q^{\left(\begin{array}{c}
k \\
2
\end{array}\right)} z^{k}
$$

сходится абсолютно при $|z|<R$. Следовательно,

$$
Q(z)=\sum_{k=0}^{\infty}\left(\sum_{t=k}^{\infty} \pi_{t}\left[\begin{array}{l}
t \\
k
\end{array}\right]_{q}\right) q^{\left(\begin{array}{c}
k \\
2
\end{array}\right)} z^{k}, \quad|z|<R,
$$

откуда вытекает (3). 
Лемма 2. Если ряд

$$
\sum_{t=0}^{\infty} a_{t} \prod_{i=0}^{t-1}\left(1+q^{i} z\right), \quad a_{t} \in \mathbf{C}, \quad t=0,1, \ldots,
$$

абсолютно сходится в точке $x>0$, то этот ряд сходится абсолютно и равномерно в любом замкнутом круге $|z| \leqslant r$, где $r<x$. В частности, сумма данного ряда являетсл аналитической в круге $|z|<x$ функцией.

Доказателъство. Утверждение леммы непосредственно следует из соотношений

$$
\begin{aligned}
& \left|a_{t}\right| x^{t} q^{\left(\begin{array}{c}
t \\
2
\end{array}\right)}<\left|a_{t}\right| x^{t} q^{\left(\begin{array}{c}
t \\
2
\end{array}\right)} \prod_{i=0}^{t-1}\left(1+q^{-i} x^{-1}\right)=\left|a_{t}\right| \prod_{i=0}^{t-1}\left(1+q^{i} x\right)<\left|a_{t}\right| x^{t} q^{\left(\begin{array}{c}
t \\
2
\end{array}\right)} \prod_{i=0}^{\infty}\left(1+q^{-i} x^{-1}\right) \\
& \left|a_{t} \prod_{i=0}^{t-1}\left(1+q^{i} z\right)\right| \leqslant\left|a_{t}\right| \prod_{i=0}^{t-1}\left(1+q^{i} r\right)
\end{aligned}
$$

справедливых при $|z| \leqslant r, t=0,1, \ldots$

Следствие 2. Ряды (2) $u \sum_{k=0}^{\infty} p_{k} q^{\left(\begin{array}{c}k \\ 2\end{array}\right)} z^{k}$ имеют одинаковые радиусы сходимости.

Следующее утверждение является частным случаем следствия 3.38 из [6].

Лемма 3. Пусть $\left\{a_{t}, t=0,1, \ldots\right\} u\left\{b_{t}, t=0,1, \ldots\right\}$ - числовые последователъности. Тогда, если для любого $t=0,1, \ldots$ выполняется соотношение

$$
a_{t}=\sum_{k=0}^{t} \frac{b_{k}}{\left(q^{-1}\right)_{t-k}}
$$

mo

$$
b_{t}=\sum_{k=0}^{t} \frac{a_{t-k} q^{-\left(\begin{array}{c}
k \\
2
\end{array}\right)(-1)^{k}}}{\left(q^{-1}\right)_{k}}
$$

для всех $t=0,1, \ldots$ Верно и обратное.

Доказательство теоремъ 1. Пусть распределение вероятностей $\left\{\pi_{k}, k=0,1, \ldots\right\}$ удовлетворяет условию (3). В силу леммы 1 при $|z|<q^{-\theta}$ имеет место (13). Полагая (13) $z=-q^{-t}, t=\theta, \theta+1, \ldots$, получим равенства

$$
Q\left(-q^{-t}\right)=\sum_{k=0}^{t} \pi_{k} \frac{\left(q^{-1}\right)_{t}}{\left(q^{-1}\right)_{t-k}}, \quad t=\theta, \theta+1, \ldots
$$

Положим

$$
b_{t}=\sum_{k=0}^{t} \frac{\pi_{k}}{\left(q^{-1}\right)_{t-k}}, \quad t=0,1, \ldots
$$

В силу (16) справедливо равенство

$$
b_{t}=\frac{Q\left(-q^{-t}\right)}{\left(q^{-1}\right)_{t}}, \quad t=\theta, \theta+1, \ldots
$$


Применяя к последовательностям $\left\{\pi_{t}, t=0,1, \ldots\right\},\left\{b_{t}, t=0,1, \ldots\right\}$ лемму 3 , получим, что

$$
\pi_{t}=\sum_{k=0}^{t} \frac{b_{t-k} q^{-\left(\begin{array}{c}
k \\
2
\end{array}\right)(-1)^{k}}}{\left(q^{-1}\right)_{k}}, \quad t=0,1, \ldots
$$

в частности,

$$
\pi_{t}=\sum_{k=0}^{\theta} \frac{b_{k} q^{-\left(\begin{array}{c}
t-k \\
2
\end{array}\right)}(-1)^{t-k}}{\left(q^{-1}\right)_{t-k}}+\sum_{k=0}^{t-\theta} \frac{b_{t-k} q^{-\left(\begin{array}{c}
k \\
2
\end{array}\right)(-1)^{k}}}{\left(q^{-1}\right)_{k}}, \quad t=\theta, \theta+1, \ldots
$$

Подставляя в (20) вместо $b_{k}, k=0,1, \ldots, \theta-1$, и $b_{t-k}, k=0,1, \ldots, t-\theta$, выражения (17) и (18) соответственно, получим (4). Непосредственно из (4) следует, что вектор (5) является решением системы неравенств (6).

Предположим теперь, что вектор $\left(\pi_{0}, \pi_{1}, \ldots, \pi_{\theta-1}\right)$ есть неотрицательное решение системы неравенств (6). Тогда все члены последовательности $\left\{\pi_{t}, t=0,1, \ldots\right\}$, определяемой с помощью соотношения (4), суть неотрицательные числа. Пусть

$$
b_{t}=\sum_{k=0}^{t} \frac{\pi_{k}}{\left(q^{-1}\right)_{t-k}}, \quad a_{t}=\sum_{k=0}^{t} \frac{p_{k}}{\left(q^{-1}\right)_{t-k}}, \cdot \quad t=0,1, \ldots
$$

Тогда (4) может быть записано в виде

$$
\pi_{t}=\sum_{k=t-\theta+1}^{t} \frac{b_{t-k} q^{-\left(\begin{array}{c}
k \\
2
\end{array}\right)}(-1)^{k}}{\left(q^{-1}\right)_{k}}+\sum_{k=0}^{t-\theta} \frac{Q\left(-q^{-(t-k)}\right) q^{-\left(\begin{array}{c}
k \\
2
\end{array}\right)}(-1)^{k}}{\left(q^{-1}\right)_{k}\left(q^{-1}\right)_{t-k}}, \quad t=\theta, \theta+1, \ldots
$$

Из определения чисел $Q_{k}, k=0,1, \ldots$, по доказанному вытекает справедливость равенств

$$
p_{t}=\sum_{k=t-\theta+1}^{t} \frac{a_{t-k} q^{-\left(\begin{array}{c}
k \\
2
\end{array}\right)}(-1)^{k}}{\left(q^{-1}\right)_{k}}+\sum_{k=0}^{t-\theta} \frac{Q\left(-q^{-(t-k)}\right) q^{-\left(\begin{array}{c}
k \\
2
\end{array}\right)(-1)^{k}}}{\left(q^{-1}\right)_{k}\left(q^{-1}\right)_{t-k}}, \quad t=\theta, \theta+1, \ldots
$$

Далее, в силу (21) и леммы 3 имеют место равенства

$$
\begin{array}{ll}
\pi_{t}=\sum_{k=0}^{t} \frac{b_{t-k} q^{-\left(\begin{array}{c}
k \\
2
\end{array}\right)(-1)^{k}}}{\left(q^{-1}\right)_{k}}, \quad t=0,1, \ldots, \\
p_{t}=\sum_{k=0}^{t} \frac{a_{t-k} q^{-\left(\begin{array}{c}
k \\
2
\end{array}\right)(-1)^{k}}}{\left(q^{-1}\right)_{k}}, \quad t=0,1, \ldots
\end{array}
$$

Из (22)-(25) следует, что для всех $t=\theta, \theta+1, \ldots$ имеет место соотношение

$$
\sum_{k=0}^{t-\theta} \frac{a_{t-k} q^{-\left(\begin{array}{c}
k \\
2
\end{array}\right)}(-1)^{k}}{\left(q^{-1}\right)_{k}}=\sum_{k=0}^{t-\theta} \frac{b_{t-k} q^{-\left(\begin{array}{c}
k \\
2
\end{array}\right)(-1)^{k}}}{\left(q^{-1}\right)_{k}}
$$

Последовательно подставляя в (26) $t=\theta, \theta+1, \ldots$, получим, что

$$
a_{t}=b_{t}, \quad t=\theta, \theta+1, \ldots
$$


По доказанному имеет место равенство (16) с заменой $\pi_{k}$ на $p_{k}$, т. е. выполняется соотношение

$$
a_{t}=\frac{Q\left(-q^{-t}\right)}{\left(q^{-1}\right)_{t}}, \quad t=\theta, \theta+1, \ldots
$$

В силу $(21),(27),(28)$, имеют место равенства

$$
Q\left(-q^{-t}\right)=b_{t}\left(q^{-1}\right)_{t}=\sum_{k=0}^{t} \frac{\pi_{k}\left(q^{-1}\right)_{t}}{\left(q^{-1}\right)_{t-k}}=\sum_{k=0}^{\infty} \pi_{k} \prod_{i=0}^{k-1}\left(1+q^{i}\left(-q^{-t}\right)\right), \quad t=\theta, \theta+1, \ldots
$$

Покажем, что функция

$$
f(z)=\sum_{k=0}^{\infty} \pi_{k} \prod_{i=0}^{k-1}\left(1+q^{i} z\right)
$$

является аналитической в круге $|z|<q^{-\theta}$. Тогда в силу (29) аналитические функции $f(z)$ и $Q(z),|z|<q^{-\theta}$, совпадают на множестве $\left\{-q^{-t}, t=\theta+1, \theta+2, \ldots\right\}$, имеющем предельную точку 0 , и, следовательно, справедливо равенство (13), из которого в силу леммы 1 вытекает, что последовательность $\left\{\pi_{k}, k=0,1, \ldots\right\}$ удовлетворяет (3).

На основании леммы 2 достаточно показать, что ряд $\sum_{k=0}^{\infty} \pi_{k} \prod_{i=0}^{k-1}\left(1+q^{i} x\right)$ сходится в каждой точке $x, 0<x<q^{-\theta}$.

В силу (22), (23) имеет место равенство

$$
\pi_{t}=p_{t}+\sum_{k=0}^{\theta-1} \frac{\left(b_{k}-a_{k}\right) q^{-\left(\begin{array}{c}
t-k \\
2
\end{array}\right)}(-1)^{k}}{\left(q^{-1}\right)_{t-k}}, \quad t=\theta, \theta+1, \ldots
$$

В соответствии с леммой 1 ряд $\sum_{t=0}^{\infty} p_{t} \prod_{i=0}^{t-1}\left(1+q^{i} x\right)$ сходится в круге $|x|<q^{-\theta}$, поэтому достаточно показать, что для любого $k=0,1, \ldots, \theta-1$ ряд $\sum_{t=\theta}^{\infty} \frac{q^{-\left(\begin{array}{c}t-k \\ 2\end{array}\right)}}{\left(q^{-1}\right)_{t-k}} \prod_{i=0}^{t-1}\left(1+q^{i} x\right)$ сходится при $0<x<q^{-\theta}$. При $x>0$ оценим сумму указанного ряда следующим образом:

$$
\begin{aligned}
\sum_{t=\theta}^{\infty} \frac{q^{-\left(\begin{array}{c}
t-k \\
2
\end{array}\right)}}{\left(q^{-1}\right)_{t-k}} \prod_{i=0}^{t-1}\left(1+q^{i} x\right) & \leqslant \sum_{t=k}^{\infty} \frac{q^{-\left(\begin{array}{c}
t-k \\
2
\end{array}\right)}}{\left(q^{-1}\right)_{t-k}} \prod_{i=0}^{t-1}\left(1+q^{i} x\right) \\
& <\prod_{i=0}^{\infty}\left(1+q^{-i} x^{-1}\right) \sum_{t=0}^{\infty} \frac{q^{-\left(\begin{array}{c}
t \\
2
\end{array}\right)} x^{t+k} q^{\left(\begin{array}{c}
t+k \\
2
\end{array}\right)}}{\left(q^{-1}\right)_{t}} \\
& =\prod_{i=0}^{\infty}\left(1+q^{-i} x^{-1}\right) q^{\left(\begin{array}{c}
k \\
2
\end{array}\right)} x^{k} \sum_{t=0}^{\infty} \frac{\left(q^{k} x\right)^{t}}{\left(q^{-1}\right)_{t}}
\end{aligned}
$$

Поскольку при $k=0,1, \ldots, \theta-1,0<x<q^{-\theta}$, справедливо неравенство $\left|q^{k} x\right|<1$, рассматриваемый ряд сходится в точке $x$, что и требовалось доказать.

Доказателъство теоремы 2. Пусть имеют место (7), (8) и распределение вероятностей $\left\{\pi_{k}, k=0,1, \ldots\right\}$ удовлетворяет (3). По теореме 1 имеют место равенства

$$
\pi_{t}=p_{t}+\sum_{l=0}^{\theta-1}\left(\pi_{l}-p_{l}\right) \sum_{k=l}^{\theta-1} \frac{q^{-\left(\begin{array}{c}
t-k \\
2
\end{array}\right)}(-1)^{t-k}}{\left(q^{-1}\right)_{t-k}\left(q^{-1}\right)_{k-l}}, \quad t=\theta, \theta+1, \ldots
$$


Положим $\varepsilon_{l}=p_{l}-\pi_{l}, l=0,1, \ldots, \theta-1$, и покажем, что $\varepsilon_{l}=0$ для всех $l=$ $0,1, \ldots, \theta-1$.

Поскольку $\pi_{t} \geqslant 0, t \in \mathbf{N}_{0}$, в силу (30) справедливо неравенство

$$
p_{t} q^{\left(\begin{array}{c}
t \\
2
\end{array}\right)} \geqslant(-1)^{t} \sum_{l=0}^{\theta-1} \varepsilon_{l} \sum_{k=l}^{\theta-1} \frac{q^{-\left(\begin{array}{c}
k+1 \\
2
\end{array}\right)}(-1)^{k} q^{t k}}{\left(q^{-1}\right)_{t-k}\left(q^{-1}\right)_{k-l}}, \quad t=\theta, \theta+1, \ldots
$$

Из (31) получаем, что

$$
p_{t} q^{\left(\begin{array}{c}
t \\
2
\end{array}\right)-(\theta-1) t} \geqslant(-1)^{t} \sum_{l=0}^{\theta-1} \varepsilon_{l} \sum_{k=l}^{\theta-1} \frac{q^{-\left(\begin{array}{c}
k+1 \\
2
\end{array}\right)}(-1)^{k} q^{-(\theta-1-k) t}}{\left(q^{-1}\right)_{t-k}\left(q^{-1}\right)_{k-l}}
$$

для всех $t=\theta, \theta+1, \ldots$

Переходя в (32) к нижнему пределу при $2 t \rightarrow \infty, 2 t+1 \rightarrow \infty$, в силу (8) получаем соотношение

$$
0=\sum_{l=0}^{\theta-1} \varepsilon_{l} \frac{q^{-\left(\begin{array}{c}
\theta \\
2
\end{array}\right)}(-1)^{\theta-1}}{\left(q^{-1}\right)_{\infty}\left(q^{-1}\right)_{\theta-1-l}}
$$

то есть

$$
\sum_{l=0}^{\theta-1} \frac{\varepsilon_{l}}{\left(q^{-1}\right)_{\theta-1-l}}=0
$$

В силу (33) имеет место равенство

$$
\sum_{l=0}^{\theta-1} \varepsilon_{l} \sum_{k=l}^{\theta-1} \frac{q^{-\left(\begin{array}{c}
k+1 \\
2
\end{array}\right)}(-1)^{k} q^{-(\theta-1-k) t}}{\left(q^{-1}\right)_{t-k}\left(q^{-1}\right)_{k-l}}=\sum_{l=0}^{\theta-2} \varepsilon_{l} \sum_{k=l}^{\theta-2} \frac{q^{-\left(\begin{array}{c}
k+1 \\
2
\end{array}\right)}(-1)^{k} q^{-(\theta-1-k) t}}{\left(q^{-1}\right)_{t-k}\left(q^{-1}\right)_{k-l}}, t=\theta, \theta+1, \ldots
$$

из которого с учетом (32) получаем неравенства

$$
p_{t} q^{\left(\begin{array}{c}
t \\
2
\end{array}\right)-(\theta-2) t} \geqslant(-1)^{t} \sum_{l=0}^{\theta-2} \varepsilon_{l} \sum_{k=l}^{\theta-2} \frac{q^{-\left(\begin{array}{c}
k+1 \\
2
\end{array}\right)}(-1)^{k} q^{-(\theta-2-k) t}}{\left(q^{-1}\right)_{t-k}\left(q^{-1}\right)_{k-l}}, \quad t=\theta, \theta+1, \ldots
$$

На основании (35) и (8) с помощью предельного перехода при $2 t, 2 t+1 \rightarrow \infty$ получим соотношения

$$
\begin{gathered}
\sum_{l=0}^{\theta-2} \frac{\varepsilon_{l}}{\left(q^{-1}\right)_{\theta-2-l}}=0, \\
p_{t} q^{\left(\begin{array}{c}
t \\
2
\end{array}\right)-(\theta-3) t} \geqslant(-1)^{t} \sum_{l=0}^{\theta-3} \varepsilon_{l} \sum_{k=l}^{\theta-3} \frac{q^{-\left(\begin{array}{c}
k+1 \\
2
\end{array}\right)(-1)^{k} q^{-(\theta-3-k) t}}}{\left(q^{-1}\right)_{t-k}\left(q^{-1}\right)_{k-l}}, \quad t=\theta, \theta+1, \ldots
\end{gathered}
$$

Продолжая аналогичные рассуждения, получим в итоге систему равенств

$$
\sum_{l=0}^{\theta--j} \frac{\varepsilon_{l}}{\left(q^{-1}\right)_{\theta-j-l}}=0, \quad j=1, \ldots, \theta
$$

из которой непосредственно следует, что $\varepsilon_{l}=0$ для всех $l=0,1, \ldots, \theta-1$.

Теорема доказана. 


\section{Список литературы}

1. Алексейчук А.Н., Об однозначности проблемы моментов в классе $q$-распределений. Дискретнал математика (1998) 10, №1, 95-110.

2. Эндрюс Г., Теория разбиений. Наука, Москва, 1982.

3. Ламбек И., Колъча и модули. Мир, Москва, 1971.

4. Алексейчук А.Н., Предельные распределения длины и ранга модуля, порожденного столбцами случайной равновероятной матрицы над конечным цепным кольцом. Кибернетика и системный анализ (1998) 5, 112-118.

5. Коваленко И.Н., Левитская А.А., Савчук М.Н., Избранные задачи вероятностной комбинаторики. Наукова думка, Киев, 1986.

6. Айгнер М., Комбинаторная теория. Мир, Москва, 1982.

Статья поступила 22.12.1998. 